MATHEMATICS OF COMPUTATION

Volume 73, Number 245, Pages 377-386

S 0025-5718(03)01528-X

Article electronically published on April 28, 2003

\title{
CLASS NUMBERS OF SOME ABELIAN EXTENSIONS OF RATIONAL FUNCTION FIELDS
}

\author{
SUNGHAN BAE, HWANYUP JUNG, AND JAEHYUN AHN
}

\begin{abstract}
Let $P$ be a monic irreducible polynomial. In this paper we generalize the determinant formula for $h\left(K_{P^{n}}^{+}\right)$of Bae and Kang and the formula for $h^{-}\left(K_{P^{n}}\right)$ of Jung and Ahn to any subfields $K$ of the cyclotomic function field $K_{P^{n}}$. By using these formulas, we calculate the class numbers $h^{-}(K), h\left(K^{+}\right)$ of all subfields $K$ of $K_{P}$ when $q$ and $\operatorname{deg}(P)$ are small.
\end{abstract}

\section{INTRODUCTION}

Let $\mathbb{A}=\mathbb{F}_{q}[T]$ be the ring of polynomials over the finite field $\mathbb{F}_{q}$ with $q$ elements, and $k=\mathbb{F}_{q}(T)$. For each polynomial $N \in \mathbb{A}$, one uses the Carlitz module $\rho$ to construct a field extension $K_{N}$, called the $N$-th cyclotomic function field, and its maximal real subfield $K_{N}^{+}$. Let $K$ be any subfield of some $K_{N}$, and $K^{+}=K \cap K_{N}^{+}$. It is well known that the divisor class number $h(K)$ of $K$ is divisible by the divisor class number $h\left(K^{+}\right)$of $K^{+}$. The relative divisor class number of $K$, denoted by $h^{-}(K)$, is given by $h(K) / h\left(K^{+}\right)$. In a recent paper Bae and Kang [BK] have obtained determinant formulas for $h^{-}\left(K_{P^{n}}\right)$ and $h\left(K_{P^{n}}^{+}\right)$, where $P$ is a monic irreducible polynomial in $\mathbb{A}$. In [JA1], Jung and Ahn have obtained a determinant formula for $h^{-}\left(K_{P^{n}}\right)$. In this paper we generalize the Bae-Kang determinant formula for $h\left(K_{P^{n}}^{+}\right)$and the Jung-Ahn formula for $h^{-}\left(K_{P^{n}}\right)$ to any subfield $K$ of $K_{P^{n}}$. Our proof is much simpler than the proofs of [BK] and [JA1].

In the classical case Girstmair $[\mathrm{G}$ generalized the Maillet determinant and expressed the relative class number $h^{-}(K)$ of an imaginary abelian number field $K$ in terms of the generalized Maillet determinant. He also computed the relative class number $h^{-}(K)$ when $K$ has a prime conductor and its degree over $\mathbb{Q}$ is small.

The layout of this paper is as follows. In section 2, we give some notation for cyclotomic extensions and their subfields of rational function fields, and we also state some earlier results needed in this paper. In section 3, we extend Kučera's lemma [Ku, Lemma 2] to the function field case. By using this lemma, we obtain determinant class number formulas for $h^{-}(K)$ and $h\left(K^{+}\right)$(Theorem 3.2). In section 4 , we consider the case of a prime conductor $P$. Since $K_{P} / k$ is a cyclic extension, all subfields of $K_{P}$ can be characterized by their degrees over $k$. We obtain reduced determinant class number formulas (Propositions 4.1 and 4.3) by using a primitive root modulo $P$. In the final section, these reduced formulas are used to calculate

Received by the editor March 27, 2002 and, in revised form, May 20, 2002.

2000 Mathematics Subject Classification. Primary 11R60, 11R29.

Key words and phrases. Class number, function field.

(C)2003 American Mathematical Society 
the class numbers $h^{-}(K)$ and $h\left(K^{+}\right)$of all subfields $K$ of $K_{P}$ when $q$ and $\operatorname{deg}(P)$ are small.

Throughout the paper, $K$ is assumed to be contained in some cyclotomic function field.

\section{BASIC FACTS AND NOTATION}

For any nonzero polynomial $A \in \mathbb{A}$, let $\operatorname{sgn}(A)$ be the leading coefficient of $A$. A nonzero polynomial $A \in \mathbb{A}$ is called monic if $\operatorname{sgn}(A)=1$. We fix a monic polynomial $N$. For any polynomial $A \in \mathbb{A}$, we denote by $\bar{A}$ the unique polynomial such that $\bar{A} \equiv A \bmod N$ and $\operatorname{deg}(\bar{A})<\operatorname{deg}(N)$. We also define $\operatorname{sgn}_{N}(A)=\operatorname{sgn}(\bar{A})$ and $\operatorname{deg}_{N}(A)=\operatorname{deg}(\bar{A})$.

It is well known that the Galois group of $K_{N}$ over $k$ is canonically isomorphic to $(\mathbb{A} / N)^{*}$. Explicitly, there is an isomorphism given as follows:

$$
\Psi:(\mathbb{A} / N)^{*} \rightarrow G_{N}, \quad A \bmod N \mapsto \sigma_{A},
$$

where $\sigma_{A}\left(\lambda_{N}\right)=\rho_{A}\left(\lambda_{N}\right)$ for any $N$-torsion element $\lambda_{N}$ of the Carlitz module. Moreover, $\Psi\left(\mathbb{F}_{q}^{*}\right)=J=\operatorname{Gal}\left(K_{N} / K_{N}^{+}\right)$. Let us denote by $\mathbb{M}_{N}$ the set of polynomials in $\mathbb{A}$ with degree less than $\operatorname{deg}(N)$ and prime to $N$, and by $\mathbb{M}_{N}^{+}$the subset of $\mathbb{M}_{N}$ consisting of monic polynomials.

For any $A \in \mathbb{M}_{N}, Z_{N}(s, A)$ denotes the partial zeta function associated to the class of $A$ in $C l(\mathbb{A})$. For $A \in \mathbb{A}$, we let $\langle A\rangle_{N}=1$ if $\operatorname{sgn}_{N}(A)=1$ and $\langle A\rangle_{N}=0$ otherwise. Then from JA2, Lemma 3.1], we have

$$
Z_{N}(0, A)=\langle A\rangle_{N}-\frac{1}{q-1} .
$$

Let $K$ be a finite abelian extension of $k$ which is contained in some cyclotomic function field. By the conductor of $K$, we mean the monic polynomial $N \in \mathbb{A}$ such that $K_{N}$ is the smallest cyclotomic function field which contains $K$. Let $K^{+}=$ $K \cap K_{N}^{+}$be the maximal real subfield of $K$. Let $G_{K}$ be the Galois group of $K / k$ and $J_{K}=\operatorname{Gal}\left(K / K^{+}\right)$. Let $\widehat{G}_{K}$ be the set of characters of $G_{K}$ with values in $\mathbb{C}$. We denote by $\chi_{0}$ the trivial character of $G_{K}$. A character $\chi \in \widehat{G}_{K}$ is called real if $\chi\left(J_{K}\right)=1$ and nonreal otherwise. Denote by $\widehat{G}_{K}^{+}$the set of all real characters of $G_{K}$, and put $\widehat{G}_{K}^{-}=\widehat{G}_{K} \backslash \widehat{G}_{K}^{+}$. Let $\mathbb{Q}\left[G_{K}\right]$ be the group ring with coefficients in $\mathbb{Q}$. For any subset $H$ of $G_{K}$, let $s(H)=\sum_{\sigma \in H} \sigma \in \mathbb{Q}\left[G_{K}\right]$. Let $e^{+}=s\left(J_{K}\right) /\left|J_{K}\right|$ and $e^{-}=1-e^{+}$. Let $\mathbb{Q}\left[G_{K}\right]^{ \pm}=\left\{x \in \mathbb{Q}\left[G_{K}\right]: e^{\mp} x=0\right\}$. There are well known class number formulas $[\mathrm{Y}, \mathrm{BK}$

$$
h^{-}(K)=\prod_{\chi \in \widehat{G}_{K}^{-}} \sum_{A \in \mathbb{M}_{N}} Z_{N}(0, A) \bar{\chi}(A)
$$

and

$$
h\left(K^{+}\right)=\prod_{\substack{\chi \in \widehat{G}_{K}^{+} \\ \chi \neq \chi_{0}}}\left(-\sum_{A \in \mathbb{M}_{N}^{+}} \operatorname{deg}(A) \chi(A)\right) .
$$

\section{Determinant Class NUmber FORMUlas}

Let $K$ be a finite abelian extension of $k$ with conductor $N=P^{n}$, a power of a monic irreducible polynomial $P$. Fix a system $C$ of representatives of $G_{K} / J_{K}$. For any $\tau \in G_{K}$, we denote by $\tilde{\tau}$ the unique element of $C$ such that $\tau \tilde{\tau}^{-1} \in J_{K}$. Let 
$\chi \in \widehat{G}_{K}$. Then $\chi$ induces a homomorphism $\mathbb{C}\left[G_{K}\right] \rightarrow \mathbb{C}$ in an obvious way. We denote it also by $\chi$.

Lemma 3.1. Let $C$ be any system of representatives of $G_{K} / J_{K}$.

(i) For any $\theta=\sum_{\sigma \in G_{K}} t_{\sigma} \sigma^{-1} \in \mathbb{Q}\left[G_{K}\right]^{-}$, we have

$$
\operatorname{det}\left(t_{\sigma \tau}-t_{\sigma \tilde{\tau}}: \sigma, \tau \notin C\right)= \pm \prod_{\chi \in \widehat{G}_{K}^{-}} \chi(\theta) .
$$

(ii) For any $\eta=\sum_{\sigma \in G_{K}} s_{\sigma} \sigma^{-1} \in \mathbb{Q}\left[G_{K}\right]^{+}$, we have

$$
\operatorname{det}\left(s_{\sigma \tau}: \sigma, \tau \in C\right)= \pm\left|J_{K}\right|^{-\left[K^{+}: k\right]} \prod_{\chi \in \widehat{G}^{+}} \chi(\eta) .
$$

Proof. For each $\chi \in \widehat{G}$, let $e_{\chi}=\frac{1}{\left|G_{K}\right|} \sum_{\sigma \in G_{K}} \chi(\sigma) \sigma^{-1}$ be the idempotent element associated to $\chi$. For (i) we consider the linear transformation $T$ of $\mathbb{C}\left[G_{K}\right]^{-}$into $\mathbb{C}\left[G_{K}\right]^{-}$defined as multiplication by $\theta$. Clearly the matrix of $T$ with respect to the basis $\left\{e_{\chi}: \chi \in \widehat{G}_{K}^{-}\right\}$is given as $\operatorname{diag}\left(\chi(\theta): \chi \in \widehat{G}_{K}^{-}\right)$. One can easily show that $X=\left\{(1-j) \sigma^{-1}: 1 \neq j \in J_{K}, \sigma \in C\right\}$ forms a basis of $\mathbb{C}\left[G_{K}\right]^{-}$. Since $C^{\prime}=\left\{\sigma^{-1}: \sigma \in C\right\}$ is also a system of representatives of $G_{K} / J_{K}$, it follows that $X^{\prime}=\left\{(1-j) \sigma: 1 \neq j \in J_{K}, \sigma \in C\right\}$ is also a basis of $\mathbb{C}\left[G_{K}\right]^{-}$. Then the matrix of $T$ from $X^{\prime}$ to $X$ is given by $\left(t_{\sigma \tau}-t_{\sigma \tilde{\tau}}: \sigma, \tau \notin C\right)$. Since $X$ and $X^{\prime}$ are bases of the free abelian group $\mathbb{Z}\left[G_{K}\right]^{-}$, the transition matrix from $X$ to $X^{\prime}$ has determinant \pm 1 , so we get (i).

For (ii) we consider the linear transformation $S$ of $\mathbb{C}\left[G_{K}\right]^{+}$into $\mathbb{C}\left[G_{K}\right]^{+}$defined by multiplication by $\eta$. Clearly $S$ has the matrix $\operatorname{diag}\left(\chi(\theta): \chi \in \widehat{G}_{K}^{+}\right)$with respect to the basis $\left\{e_{\chi}: \chi \in \widehat{G}_{K}^{+}\right\}$. It is easy to show that $Y=\left\{s\left(J_{K}\right) \sigma^{-1}: \sigma \in C\right\}$ and $Y^{\prime}=\left\{s\left(J_{K}\right) \sigma: \sigma \in C\right\}$ constitute bases of $\mathbb{Q}\left[G_{K}\right]^{+}$, and the matrix of $S$ from $Y^{\prime}$ to $Y$ is given by $\left(\left|J_{K}\right| s_{\sigma \tau}: \sigma, \tau \in C\right)$. Since $Y$ and $Y^{\prime}$ are also bases of the free abelian group $\mathbb{Z}\left[G_{K}\right]^{+}$, the transition matrix from $Y$ to $Y^{\prime}$ has determinant \pm 1 , so we get (ii).

For $A \in \mathbb{M}_{N}$, let $\sigma_{A}^{\prime}$ be the restriction of $\sigma_{A}$ to $K$. By using Lemma 3.1 and the class number formulas (2.2) and (2.3), we obtain the following determinant class number formulas.

Theorem 3.2. Let $K / k$ be any finite abelian extension with conductor $N=P^{n}$. Let $C$ be any system of representatives of $G_{K} / J_{K}$.

(i) For $\sigma \in G_{K}$, let $t_{\sigma}=\sum_{A \in \mathbb{M}_{N}, \sigma_{A}^{\prime}=\sigma} Z_{N}(0, A)$. Then

$$
h^{-}(K)=\left|\operatorname{det}\left(t_{\sigma \tau}-t_{\sigma \tilde{\tau}}: \sigma, \tau \notin C\right)\right| .
$$

(ii) For $\sigma \in G_{K}$, let $s_{\sigma}=-\frac{\left|J_{K}\right|}{q-1} \sum_{A \in \mathbb{M}_{N}, \sigma_{A}^{\prime}=\sigma} \operatorname{deg}(A)$. Then

$$
h\left(K^{+}\right)=\frac{1}{d(N)}\left|\operatorname{det}\left(s_{\sigma \tau}: \sigma, \tau \in C\right)\right|,
$$

where $d(N)=\sum_{A \in \mathbb{M}_{N}^{+}} \operatorname{deg}(A)$.

Proof. (i) Take

$$
\theta=\sum_{\sigma \in G_{K}}\left(\sum_{A \in \mathbb{M}_{N}, \sigma_{A}^{\prime}=\sigma} Z_{N}(0, A)\right) \sigma^{-1}
$$


We note that $\theta \in \mathbb{Q}\left[G_{K}\right]^{-}$and $\chi(\sigma)=\chi(A)$ for any $A \in \mathbb{M}_{N}$ with $\sigma_{A}^{\prime}=\sigma$. Thus for any $\chi \in \widehat{G}_{K}^{-}$, we have

$$
\chi(\theta)=\sum_{\sigma \in G_{K}} \bar{\chi}(\sigma) \sum_{\substack{A \in \mathbb{M}_{N} \\ \sigma_{A}^{\prime}=\sigma}} Z_{N}(0, A)=\sum_{A \in \mathbb{M}_{N}} Z_{N}(0, A) \bar{\chi}(A) .
$$

By Lemma 3.1(i) and the class number formula (2.2), we get (3.3).

(ii) Take $\eta=\sum_{\sigma \in G_{K}} s_{\sigma} \sigma^{-1}$ with $s_{\sigma}=-\frac{\left|J_{K}\right|}{q-1} \sum_{A \in \mathbb{M}_{N}, \sigma_{A}^{\prime}=\sigma} \operatorname{deg}(A)$. For any $\chi \in \widehat{G}_{K}^{+}$, we have

$$
\begin{aligned}
\chi(\theta) & =-\frac{\left|J_{K}\right|}{q-1} \sum_{\sigma \in G_{K}} \bar{\chi}(\sigma) \sum_{\substack{A \in \mathbb{M}_{N} \\
\sigma_{A}^{\prime}=\sigma}} \operatorname{deg}(A) \\
& =-\frac{\left|J_{K}\right|}{q-1} \sum_{A \in \mathbb{M}_{N}} \operatorname{deg}(A) \bar{\chi}(A)=-\left|J_{K}\right| \sum_{A \in \mathbb{M}_{N}^{+}} \operatorname{deg}(A) \bar{\chi}(A) .
\end{aligned}
$$

In particular, if $\chi=\chi_{0}$, then $\chi(\theta)=-\left|J_{K}\right| d(N)$. Thus by Lemma 3.1 (ii) and the class number formula (2.3), we get (3.4).

Remark 3.3. (i) One can easily see that Proposition 3.2 (i) and (ii) are generalizations of [JA1, Proposition 2.5] and [BK] Theorem 4], respectively.

(ii) Let $\bar{t}_{\sigma}=\sum_{A \in \mathbb{M}_{N}, \sigma_{A}^{\prime}=\sigma}\langle A\rangle_{N}$. Then by (2.1), Theorem 3.2 can be read as

$$
h^{-}(K)=\left|\operatorname{det}\left(\bar{t}_{\sigma \tau}-\bar{t}_{\sigma \tilde{\tau}}: \sigma, \tau \notin C\right)\right| .
$$

\section{THE CASE OF PRIME CONDUCTOR}

In this section we assume that $K$ has the conductor $P$, where $P$ is a monic irreducible polynomial of degree $d$ in $\mathbb{A}$. Let $n=[K: k], n_{0}=\left[K^{+}: k\right]$. Choose a primitive root $Q$ modulo $P$. Clearly we may assume that $\operatorname{deg}(Q)<\operatorname{deg}(P)$. For each $i \in \mathbb{Z}$, let $Q_{i} \in \mathbb{M}_{P}$ be the unique element such that $Q_{i} \equiv Q^{i} \bmod P$. Thus $Q_{i}=Q_{i^{\prime}}$ if $i \equiv i^{\prime} \bmod \left(q^{d}-1\right)$. Let $\sigma \in G_{K}$ be the restriction of $\sigma_{Q} \in G_{P}$ to $K$. Then $G_{K}$ is a cyclic group of order $n$ generated by $\sigma$. Since $\operatorname{Gal}\left(K_{P} / K\right)=$ $\left\{\sigma_{Q_{n j}}: 0 \leq j \leq \frac{q^{d}-1-n}{n}\right\}$ and $\operatorname{Gal}\left(K_{P} / K^{+}\right)=\left\{\sigma_{Q_{n_{0} j}}: 0 \leq j \leq \frac{q^{d}-1-n_{0}}{n_{0}}\right\}$, the sign group $J_{K}$ is generated by $\sigma^{n_{0}}$. Thus $C=\left\{\sigma^{i}: 0 \leq i \leq n_{0}-1\right\}$ is a system of representatives of $G_{K} / J_{K}$. For each $i \in \mathbb{Z}$, we define $i^{*}$ to be the unique integer such that $i \equiv i^{*} \bmod n_{0}$ and $0 \leq i^{*}<n_{0}$. Then $\widetilde{\sigma^{i}}=\sigma^{i^{*}}$. For each $i \in \mathbb{Z}$, we let $t_{i}=t_{\sigma^{i}}$ and $s_{i}=s_{\sigma^{i}}$. Clearly $t_{i}=t_{i^{\prime}}, s_{i}=s_{i^{\prime}}$ if $i \equiv i^{\prime} \bmod n$. Then we have

$$
t_{i}=\sum_{j} Z_{P}\left(0, Q_{i+j n}\right), \quad s_{i}=-\frac{\left|J_{K}\right|}{q-1} \sum_{j} \operatorname{deg}\left(Q_{i+j n}\right),
$$

where $0 \leq j \leq \frac{q^{d}-1-n}{n}$. We note that by (2.1) we have

$$
t_{i}=\sum_{j}\left\langle Q_{i+j n}\right\rangle_{P}-\left(q^{d}-1\right) / n(q-1) .
$$

In this case Theorem 3.2 can be written as follows. 
Proposition 4.1. Let $K / k$ be a finite abelian extension with prime conductor $P$. With the same notation as above, we have the following class numbers formulas:

(i) $h^{-}(K)=\left|\operatorname{det}\left(t_{i+j}-t_{i+j^{*}}: n_{0} \leq i, j \leq n-1\right)\right|$,

(ii) $h\left(K^{+}\right)=\frac{1}{d(P)}\left|\operatorname{det}\left(s_{i+j}: 0 \leq i, j \leq n_{0}-1\right)\right|$.

Since $\operatorname{Gal}\left(K_{P} / K_{P}^{+}\right)=\left\langle\sigma_{Q}^{\left(q^{d}-1\right) /(q-1)}\right\rangle$ and $\operatorname{Gal}\left(K_{P} / K\right)=\left\langle\sigma_{Q}^{n}\right\rangle$, we have

$$
\operatorname{Gal}\left(K_{P} / K^{+}\right)=\left\langle\sigma_{Q}^{q^{d}-1 / q-1}, \sigma_{Q}^{n}\right\rangle
$$

Thus $n_{0}=\operatorname{gcd}\left(n, \frac{q^{d}-1}{q-1}\right)$. Since $K_{P} / k$ is a cyclic extension, we can completely determine all subfields, their sign groups and their class numbers by Proposition 4.1 .

Lemma 4.2. For each $i, j \in\{0, \ldots, n-1\}$, we have

(i) $\sum_{0 \leq e \leq\left(n / n_{0}\right)-1} t_{i+e n_{0}}=0$,

(ii) $s_{i}=s_{i^{\prime}}$ if $i \equiv i^{\prime} \bmod n_{0}$,

(iii) $s_{0}+\ldots+s_{n_{0}-1}=-d(P)$.

Proof. Let $S_{i}=\left\{Q_{i+j n}: j=0, \ldots, \frac{q^{d}-1-n}{n}\right\}$ for $i=0, \ldots, n-1$. Then $\left\{S_{i}\right.$ : $i=0, \ldots, n-1\}$ forms a partition of $\mathbb{M}_{P}^{n}$. It is easy to see that if $\sigma_{A}^{\prime}=\sigma^{e}$ for some $A \in \mathbb{M}_{P}$, then multiplication by $A$ followed by reduction modulo $P$ defines a one-to-one map from $S_{i}$ to $S_{i+e}$ for each $i$. Suppose that $Z_{P}(0, A)$ appears in the summation of (i) with nonzero coefficient. Since $J_{K}$ is generated by $\sigma^{n_{0}}$ and $\operatorname{res}_{K_{P} / K}\left(\operatorname{Gal}\left(K_{P} / K_{P}^{+}\right)\right)=J_{K}$, for any $\alpha \in \mathbb{F}_{q}^{*}$ we have $\sigma_{\alpha}^{\prime}=\sigma^{e n_{0}}$, and so $Z_{P}(0, \alpha A)$ also appears in the summation of (i) exactly once. Since $\sum_{\alpha \in \mathbb{F}_{q}^{*}} Z_{P}(0, \alpha A)=0$ by (2.1), we get (i). Suppose that (ii) is true. Then we have

$$
\begin{aligned}
s_{0}+\ldots+s_{n_{0}-1} & =-\frac{\left|J_{K}\right|}{q-1} \sum_{0 \leq i \leq n_{0}-1} \sum_{0 \leq j \leq \frac{q^{d}-1-n}{n}} \operatorname{deg}\left(Q_{i+j n}\right) \\
& =-\frac{1}{q-1} \sum_{0 \leq i \leq n-1} \sum_{0 \leq j \leq \frac{q^{d}-1-n}{n}} \operatorname{deg}\left(Q_{i+j n}\right)=-d(P),
\end{aligned}
$$

so we get (iii). It remains to prove (ii). Suppose that $i^{\prime}=i+e n_{0}$ for some $e$. Then there exists $\alpha \in \mathbb{F}_{q}^{*}$ with $\sigma_{\alpha}^{\prime}=\sigma^{e n_{0}}$, and so we have

$$
s_{i^{\prime}}=-\frac{\left|J_{K}\right|}{q-1} \sum_{j} \operatorname{deg}\left(\alpha Q_{i+j n}\right)=s_{i} .
$$

Now we give reduced matrices of the matrices given in Proposition 4.1 Define

$$
\mathbb{S}_{K}=\left(\begin{array}{cccccc}
1 & 1 & 1 & \ldots & 1 & 1 \\
s_{0} & s_{1} & s_{2} & \ldots & s_{n_{0}-2} & s_{n_{0}-1} \\
\vdots & \vdots & \vdots & & \vdots & \vdots \\
s_{n_{0}-2} & s_{n_{0}-1} & s_{0} & \ldots & s_{n_{0}-4} & s_{n_{0}-3}
\end{array}\right) .
$$

Proposition 4.3. (i) $h^{-}(K)=\left|J_{K}\right|^{\left[K^{+}: k\right]}\left|\operatorname{det}\left(t_{i+j}: n_{0} \leq i, j \leq n-1\right)\right|$.

(ii) $h\left(K^{+}\right)=\left|\operatorname{det}\left(\mathbb{S}_{K}\right)\right|$. 
Proof. Consider the matrix $\left(t_{i+j}-t_{i+j^{*}}: n_{0} \leq i, j \leq n-1\right)$ in Proposition 4.1(i). For each $0 \leq j^{*} \leq n_{0}-1$, add all $\left(j^{*}+u n_{0}\right)$-columns with $u=2, \ldots,\left(n / n_{0}\right)-1$ to the $\left(j^{*}+n_{0}\right)$-column. Then the $\left(i, j^{*}+n_{0}\right)$-entry becomes

$$
\begin{aligned}
& \sum_{1 \leq u \leq\left(n / n_{0}\right)-1} t_{i+j^{*}+u n_{0}}-\left(\left(n / n_{0}\right)-1\right) t_{i+j^{*}} \\
& =\sum_{0 \leq u \leq\left(n / n_{0}\right)-1} t_{i+j^{*}+u n_{0}}-\left(n / n_{0}\right) t_{i+j^{*}} \\
& =-\left(n / n_{0}\right) t_{i+j^{*}}
\end{aligned}
$$

by Lemma 4.2 (i). By using Lemma 4.2 (i) again, we get (i). From Lemma 4.2 (ii), (iii) and elementary row operations, we can change the matrix in Proposition 4.1 (ii) to

$$
\left(\begin{array}{cccccc}
-d(P) & -d(P) & -d(P) & \ldots & -d(P) & -d(P) \\
s_{0} & s_{1} & s_{2} & \ldots & s_{n_{0}-2} & s_{n_{0}-1} \\
\vdots & \vdots & \vdots & & \vdots & \vdots \\
s_{n_{0}-2} & s_{n_{0}-1} & s_{0} & \ldots & s_{n_{0}-4} & s_{n_{0}-3}
\end{array}\right) .
$$

Thus we get (ii).

Remark 4.4. Let $\mathcal{O}_{K}$ be the integral closure of $\mathbb{A}$ in $K$ and $\mathcal{O}_{K}^{*}$ the unit group of $\mathcal{O}_{K}$. Let $h\left(\mathcal{O}_{K}\right)$ and $h\left(\mathcal{O}_{K^{+}}\right)$be the ideal class numbers of $\mathcal{O}_{K}$ and $\mathcal{O}_{K^{+}}$, and let $h^{-}\left(\mathcal{O}_{K}\right)=h\left(\mathcal{O}_{K}\right) / h\left(\mathcal{O}_{K^{+}}\right)$be the relative ideal class number of $\mathcal{O}_{K}$. Then from BJA, (2.1)], we have $h^{-}(K)=\left|J_{K}\right|^{\left[K^{+}: k\right]-1} h^{-}\left(\mathcal{O}_{K}\right)$. Thus by Proposition 4.3

$$
h^{-}\left(\mathcal{O}_{K}\right)=\left|J_{K}\right| \cdot\left|\operatorname{det}\left(t_{i+j}: n_{0} \leq i, j \leq n-1\right)\right| .
$$

Let $\ell$ be a prime divisor of $q-1$. We consider the subfields $K$ of $K_{P}$ of degree $\ell$ over $k$, which are characterized as follows [An Lemma 3.2].

Lemma 4.5. Let $K / k$ be a finite abelian extension with prime conductor $P$, whose degree over $k$ is $\ell$.

(i) If $d \equiv 0 \bmod \ell$. Then $K=k(\sqrt[\ell]{P})$ and it is contained in $K_{P}^{+}$.

(ii) If $d \not \equiv 0 \bmod \ell$, let $e, 1 \leq e \leq \ell-1$, be such that $e d \equiv 1 \bmod \ell$. Then $K=k\left(\sqrt[\ell]{-P^{e}}\right)$ and it is not contained in $K_{P}^{+}$.

We calculate the class number $h(K)$ by using Proposition 4.3. We note that if $d \not \equiv 0 \bmod \ell$, then $K^{+}=k, G_{K}=J_{K}, h(K)=h^{-}(K)$, and if $d \equiv 0 \bmod \ell$, then $K^{+}=K, J_{K}=\{1\}, h(K)=h\left(K^{+}\right)$. Define

$$
\mathbb{D}_{K}=\left(\begin{array}{cccccc}
t_{0} & t_{1} & t_{2} & \ldots & t_{\ell-3} & t_{\ell-2} \\
t_{1} & t_{2} & t_{3} & \ldots & t_{\ell-2} & t_{\ell-1} \\
\vdots & \vdots & \vdots & & \vdots & \vdots \\
t_{\ell-2} & t_{\ell-1} & t_{0} & \ldots & t_{\ell-5} & t_{\ell-4}
\end{array}\right), \quad \text { if } d \not \equiv 0 \bmod \ell,
$$

and $\mathbb{D}_{K}=\mathbb{S}_{K}$ if $d \equiv 0 \bmod \ell$.

Corollary 4.6. We let $\varepsilon=1$ if $d \not \equiv 0 \bmod \ell$, and $\varepsilon=0$ otherwise. Then $h(K)=$ $\ell^{\varepsilon}\left|\operatorname{det}\left(\mathbb{D}_{K}\right)\right|$. 
Proof. We only prove the case that $d \not \equiv 0 \bmod \ell$. By using the fact that $\sum_{i=0}^{\ell-1} t_{i}=$ $\sum_{A \in \mathbb{M}_{N}} Z_{N}(0, A)=0$ and by elementary row operations, we can change the matrix $\left(t_{i+j}: 1 \leq i, j \leq \ell-1\right)$ in Proposition 4.3 (i) to $\mathbb{D}_{K}$ up to \pm 1 . Thus we get the result.

Assume that $q$ is odd. Then the class number of the quadratic subfield of $K_{P}$ can be obtained in a simple form as follows.

Corollary 4.7. Let $q$ be odd. Let $X_{0}$ and $X_{1}$ be the subsets of $\mathbb{M}_{P}$ consisting of quadratic residues modulo $P$ and quadratic nonresidues modulo $P$, respectively.

(i) If $\operatorname{deg}(P)$ is odd, then the class number of $k(\sqrt{-P})$ is

$$
\left|2 S-\frac{q^{d}-1}{q-1}\right|
$$

where $S=\left|X_{0} \cap \mathbb{M}_{P}^{+}\right|$.

(ii) If $\operatorname{deg}(P)$ is even, then the class number of $k(\sqrt{P})$ is

$$
\frac{1}{q-1}\left|(q-1) d(P)-2 \sum_{A \in X_{0}} \operatorname{deg}(A)\right|
$$

In particular, if $\operatorname{deg}(P)=2$, then $h(k(\sqrt{P}))=1$.

Proof. Let $K$ be the unique quadratic subfield of $K_{P}$. Assume that $d \not \equiv 0 \bmod 2$. Note that for any $A \in \mathbb{M}_{P}, \sigma_{A}^{\prime}=1$ if and only if $A$ is a quadratic residue modulo $P$. Then by Corollary 4.6 we have $h(K)=2\left|t_{0}\right|$, where $t_{0}=\sum_{A \in \mathbb{M}_{P} \cap X_{0}} Z_{P}(0, A)$. By the definition of $S$, we have $t_{0}=S-\frac{q^{d}-1}{2(q-1)}$, which implies (i).

Now assume that $d \equiv 0 \bmod 2$. Then by Lemma 4.2 (iii) and Corollary 4.6, we have $h(K)=\left|s_{0}-s_{1}\right|=\left|2 s_{0}+d(P)\right|$. Since

$$
s_{0}=-\frac{1}{q-1} \sum_{1 \leq j \leq \frac{q^{d}-3}{2}} \operatorname{deg}\left(Q_{2 j}\right)=-\frac{1}{q-1} \sum_{A \in X_{0}} \operatorname{deg}(A) \text {, }
$$

we get (ii).

\section{Some Numerical EXAMPLES}

Let $K / k$ be a finite abelian extension with conductor $P$. In this section, we give some numerical examples for the class numbers $h^{-}(K)$ and $h\left(K^{+}\right)$of $K$ using Proposition 4.3. Here we used MAPLE for the computation. As the $k$-isomorphisms $T \mapsto T+\alpha$ with $\alpha \in \mathbb{F}_{q}^{*}$ send a monic irreducible polynomial to another monic irreducible polynomial, it suffices to consider only the monic irreducible polynomials up to these $k$-isomorphisms. In $[\mathrm{Ar}]$, Artin calculated the class number of quadratic function fields. One can see that our numerical data coincide with Artin's $\mathrm{Ar} p$. $80-81]$ in the real quadratic function field case.

Example 5.1. Assume $q=3$. There is only one monic irreducible polynomial $T$ of degree 1 up to the above $k$-isomorphisms. It is already known that $h^{-}\left(K_{T}\right)=$ $h\left(K_{T}^{+}\right)=1$. 
TABLE 1. $q=3, \operatorname{deg} P=2$

\begin{tabular}{|c|c|c|c|c|}
\hline$P$ & {$[K: k]$} & {$\left[K^{+}: k\right]$} & $h^{-}(K)$ & $h\left(K^{+}\right)$ \\
\hline \multirow{3}{*}{$T^{2}+1$} & 2 & 2 & 1 & 1 \\
\cline { 2 - 5 } & 4 & 4 & 1 & 1 \\
\cline { 2 - 5 } & 8 & 4 & $2^{3}$ & 1 \\
\hline
\end{tabular}

TABLE 2. $q=3, \operatorname{deg} P=3$

\begin{tabular}{|c|c|c|c|c|}
\hline$P$ & {$[K: k]$} & {$\left[K^{+}: k\right]$} & $h^{-}(K)$ & $h\left(K^{+}\right)$ \\
\hline \multirow{3}{*}{$T^{3}+2 T+1$} & 2 & 1 & 1 & 1 \\
\cline { 2 - 5 } & 13 & 13 & 1 & $3^{9}$ \\
\cline { 2 - 5 } & 26 & 13 & $2^{12} \cdot 3^{6}$ & $3^{9}$ \\
\hline \multirow{3}{*}{$T^{3}+2 T+2$} & 2 & 1 & 7 & 1 \\
\cline { 2 - 5 } & 13 & 13 & 1 & $3^{9}$ \\
\cline { 2 - 5 } & 26 & 13 & $2^{12} \cdot 3^{3} \cdot 3^{7}$ & $3^{9}$ \\
\hline \multirow{3}{*}{$T^{3}+T^{2}+2$} & 2 & 1 & 5 & 1 \\
\cline { 2 - 5 } & 13 & 13 & 1 & $53 \cdot 313$ \\
\cline { 2 - 5 } & 26 & 13 & $2^{12} \cdot 5 \cdot 79$ & $53 \cdot 313$ \\
\hline \multirow{3}{*}{$T^{3}+2 T^{2}+1$} & 2 & 1 & 3 & 1 \\
\cline { 2 - 5 } & 13 & 13 & 1 & $53 \cdot 313$ \\
\cline { 2 - 5 } & 26 & 13 & $2^{12} \cdot 3 \cdot 131$ & $53 \cdot 313$ \\
\hline
\end{tabular}

TABle $3 . q=3, P=T^{4}+T+2$

\begin{tabular}{|c|c|c|c|c|}
\hline$P$ & {$[K: k]$} & {$\left[K^{+}: k\right]$} & $h^{-}(K)$ & $h\left(K^{+}\right)$ \\
\hline \multirow{9}{*}{$T^{4}+T+2$} & 2 & 2 & 1 & $2^{2}$ \\
\hline & 4 & 4 & 1 & $2^{3} \cdot 17$ \\
\hline & 5 & 5 & 1 & 71 \\
\hline & 8 & 8 & 1 & $2^{4} \cdot 17 \cdot 97$ \\
\hline & 10 & 10 & 1 & $2^{2} \cdot 71 \cdot 491$ \\
\hline & 16 & 8 & $2^{7} \cdot 881$ & $2^{4} \cdot 17 \cdot 97$ \\
\hline & 20 & 20 & 1 & $2^{3} \cdot 11^{2} \cdot 17 \cdot 71 \cdot 491 \cdot 541$ \\
\hline & 40 & 40 & 1 & $\begin{array}{c}2^{4} \cdot 11^{2} \cdot 17 \cdot 41 \cdot 71 \cdot 97 \cdot 491 \cdot 541 \\
\cdot 881 \cdot 1564361 \\
\end{array}$ \\
\hline & 80 & 40 & $\begin{array}{c}2^{39} \cdot 241 \cdot 641 \cdot 881 \\
.532611841\end{array}$ & $\begin{array}{c}2^{4} \cdot 11^{2} \cdot 17 \cdot 41 \cdot 71 \cdot 97 \cdot 491 \cdot 541 \\
\cdot 881 \cdot 1564361\end{array}$ \\
\hline
\end{tabular}

There is also one monic irreducible polynomial $T^{2}+1$ of degree 2 up to the above $k$-isomorphisms. Table 1 gives the degrees $[K: k],\left[K^{+}: k\right]$ and class numbers $h^{-}(K), h\left(K^{+}\right)$for all subfields $K$ of $K_{T^{2}+1}$.

There are four monic irreducible polynomials $T^{3}+2 T+1, T^{3}+2 T+2, T^{3}+T^{2}+2$ and $T^{3}+2 T^{2}+1$ of degree 3 up to the above $k$-isomorphisms. The table for these polynomials are given in Table 2 .

There are six monic irreducible polynomials of degree 4 up to the above $k$ isomorphisms. We only give the table for $T^{4}+T+2$ (Table 3 ). 
TABle 4. $q=5, P=T^{2}+2$

\begin{tabular}{|c|c|c|c|c|}
\hline$P$ & {$[K: k]$} & {$\left[K^{+}: k\right]$} & $h^{-}(K)$ & $h\left(K^{+}\right)$ \\
\hline \multirow{5}{*}{$T^{2}+2$} & 2 & 2 & 1 & 1 \\
\cline { 2 - 5 } & 3 & 3 & 1 & 1 \\
\cline { 2 - 5 } & 4 & 2 & $2 \cdot 5$ & 1 \\
\cline { 2 - 5 } & 6 & 6 & 1 & 1 \\
\cline { 2 - 5 } & 8 & 2 & $2^{2} \cdot 5 \cdot 17$ & 1 \\
\cline { 2 - 5 } & 12 & 6 & $2^{5} \cdot 5$ & 1 \\
\cline { 2 - 5 } & 24 & 6 & $2^{10} \cdot 5 \cdot 7^{2} \cdot 17$ & 1 \\
\hline
\end{tabular}

Example 5.2. Assume $q=5$. For $P=T^{2}+2$, it is easy to check that $Q=T+1$ is a primitive root modulo $T^{2}+2$. Note that $\left[K_{P}: k\right]=24$. We consider the unique subfield $K$ of $K_{P}$ with degree $[K: k]=12$. Then $\left[K^{+}: k\right]=6$ and $\left|J_{K}\right|=2$. The matrix $\left(t_{i+j}: n_{0} \leq i, j \leq n-1\right)$ in Proposition 4.3 becomes

$$
\left(\begin{array}{cccccc}
\frac{1}{2} & \frac{1}{2} & \frac{-1}{2} & \frac{1}{2} & \frac{1}{2} & \frac{1}{2} \\
\frac{1}{2} & \frac{-1}{2} & \frac{1}{2} & \frac{1}{2} & \frac{1}{2} & \frac{-1}{2} \\
\frac{-1}{2} & \frac{1}{2} & \frac{1}{2} & \frac{1}{2} & \frac{-1}{2} & \frac{-1}{2} \\
\frac{1}{2} & \frac{1}{2} & \frac{1}{2} & \frac{-1}{2} & \frac{-1}{2} & \frac{1}{2} \\
\frac{1}{2} & \frac{1}{2} & \frac{-1}{2} & \frac{-1}{2} & \frac{1}{2} & \frac{-1}{2} \\
\frac{1}{2} & \frac{-1}{2} & \frac{-1}{2} & \frac{1}{2} & \frac{-1}{2} & \frac{-1}{2}
\end{array}\right)
$$

Then we have $h^{-}(K)=2^{5} \cdot 5$ and $h^{-}\left(\mathcal{O}_{K}\right)=5$. The matrix $\mathbb{S}_{K}$ in Proposition 4.3 becomes

$$
\left(\begin{array}{rrrrrr}
1 & 1 & 1 & 1 & 1 & 1 \\
0 & -1 & -1 & -1 & -1 & -1 \\
-1 & -1 & -1 & -1 & -1 & 0 \\
-1 & -1 & -1 & -1 & 0 & -1 \\
-1 & -1 & -1 & 0 & -1 & -1 \\
-1 & -1 & 0 & -1 & -1 & -1
\end{array}\right) .
$$

Thus we have $h\left(K^{+}\right)=1$. Moreover, the table for $T^{2}+2$ is Table 4 .

\section{REFERENCES}

[An] B. Angles, On Hilbert class field towers of global function fields. Drinfeld modules, modular schemes and applications (Alden-Biesen) World Sci. Publishing, River Edge, NJ (1997), 261-271. MR 99g:11133

[Ar] E. Artin, The collected papers of Emil Artin. Edited by Serge Lang and John T. Tate Addison-Wesley Publishing Co., Inc., Reading, Mass.-London 1965. MR 31:1159

[BJA] S. Bae, H. Jung and J. Ahn, Cyclotomic units and Stickelberger ideals of global function fields, to appear in Trans. Amer. Math. Soc.

[BK] S. Bae and P. Kang, Class numbers of cyclotomic function fields. Acta Arith. 102 (2002), no. 3, 251-259.

[G] K. Girstmair, The relative class numbers of imaginary cyclic fields of degrees 4, 6, 8, and 10. Math. Comp. 61 (1993), no. 204, 881-887. MR 94a:11170 
[JA1] H. Jung and J. Ahn, On the relative class number of cyclotomic function fields. Acta Arith. 107 (2003), no. 1, 91-101.

[JA2] H. Jung and J. Ahn, Demjanenko matrix and recursion formula for relative class number over function fields. J. Number Theory 98 (2003), no. 1, 55-66.

$[\mathrm{Ku}] \quad \mathrm{R}$. Kučera, Formulae for the relative class number of an imaginary abelian field in the form of a determinant. Nagoya Math. J. 163 (2001), 167-191. MR 2002j:11129

[Y] L. Yin, Stickelberger ideals and relative class numbers in function fields. J. Number Theory. 81 (2000), no. 1, 162-169. MR 2001d:11114

Department of Mathematics, KAist, Daejon, 305-701 Korea

E-mail address: shbae@math.kaist.ac.kr

Department of Mathematics, KAist, Daejon, 305-701 Korea

E-mail address: hyjung@mathx.kaist.ac.kr

Department of Mathematics, KAist, Daejon, 305-701 Korea

E-mail address: jaehyun@mathx.kaist.ac.kr 